\section{Reply to Lavi \& Sapir (2015): floral colour and pollinator-mediated selection in Oncocyclus irises (Iridaceae)}

In a recent research article published in New Phytologist, Lavi \& Sapir (2015) investigated the adaptive significance of the large and dark flowers of irises belonging to the section Oncocyclus (genus Iris, family Iridaceae). These plants are strictly endemic to dry, Mediterranean-type climates, particularly to the semi-desert areas of the Middle East, Turkey and the Caucasus (Mathew, 1989). Using a selection gradients approach, Lavi \& Sapir (2015) statistically tested the significance of flower size, stem length and floral colour on plant fitness in two Oncocyclus species (Iris atropurpurea Baker and I. haynei Baker). The authors used a standard approach by comparing the seed set of open-pollinated flowers to flowers receiving supplementary hand pollination to control for the effect of pollen limitation on plant fitness, thereby discriminating between pollinator-mediated selection on phenotypic traits and other sources of selection.

Regression techniques have been used since the early 1980 s to estimate the direction and magnitude of selection (Lande \& Arnold, 1983), and it is now clear that measures of long-run selection at higher taxonomical levels (here, a group of Iris species) require a more thorough understanding of the selection processes, for example, by attempting to disentangle the role of environmental drivers (climate, fires, floods, etc.) on the strength of selection on floral traits (Rausher, 1992). In their recent work, Lavi \& Sapir (2015, p. 369) used a single site (albeit the largest known) for each study species to conclude that 'phenotypic color variation in these irises is neutral'.

Countless authors have discussed the role of floral colours in floral biology and pollinator behaviour. Since the advent of modern spectrophotometry techniques and progress in uncovering the electrophysiological bases of insect vision, it has become clear that 'subjective human judgements of flower colour may be inaccurate and irrelevant to the ecology and vision of the flower's pollinators' (Arnold et al., 2010, p. 1). In their article Lavi \& Sapir (2015) measured the concentration of a single pigment (anthocyanin) in outer petal extracts of the irises by spectrophotometry at a single wavelength $(530 \mathrm{~nm})$ under laboratory conditions as a rough proxy for flower colour. The authors then interpreted the lack of correlation between anthocyanin concentration and female reproductive success as evidence for the absence of pollinator-mediated selection on floral colours in the Oncocyclus irises. By restricting their analytical approach to the quantification of anthocyanin concentration, Lavi \& Sapir (2015) failed to take into account other important aspects of floral colour that drive changes in pollinator behaviour, such as the presence of co-pigments or other pigments (that influence floral hue, see e.g. Hopkins \& Rausher, 2012; Sobel \& Streisfeld, 2013) or other surface effects (such as epidermal cell shape and structure, see e.g. Papiorek et al., 2014). Integrating knowledge of insect vision, including the patterns of photoreceptor excitation in trichromatic bees (the pollinators of these irises), as well as the effect of background colour on visual discrimination capabilities of different floral colours by bees (Arnold et al., 2010) and results from other recent studies would have been beneficial to the discussion of the adaptive value of floral colours as perceived by the pollinators. Several colour space models such as the bee hexagon (Chittka, 1992) have been developed over the past two decades to depict how the spectral reflectance of flowers whose colour appear distinct to a human can look similar to a pollinator, and vice versa. These models (Chittka \& Raine, 2006), have the advantages of first, allowing predictions on the bee-subjective appearance of object colours relative to their environmental background and second, in presenting this information in a quantitative graphical format. This approach is essential to interpret the perception of floral reflectance in its ecological context using photoreceptor sensitivities of specific pollinators, and was not used in the article by Lavi \& Sapir (2015).

Lavi \& Sapir's (2015) approach to the study of pollinatormediated selection on floral colours is in contrast with our recent studies, where we have used a range of state-of-the-art methods for the analysis of floral colours and scents from a pollinator perspective and in a phylogenetic context (with reconstructions of ancestral pollination strategies). Indeed, our recent results on the role of floral colours and scents in the parallel evolution of sheltermimicking flowers in the Orchidaceae (genera Ophrys and Serapias), and in the Iridaceae (section Oncocyclus) clearly demonstrate that virtually all extant shelter-mimicking plant species share the same suite of morphological traits, floral scent compounds and spectral patterns (such as 'colour') as adaptations to selectively attract their shared narrow taxonomical guild of specialized pollinators, primarily sheltering male bees (Vereecken et al., 2012). Furthermore, we also showed that the flowers of Iris atropurpurea in particular (a species investigated by Lavi \& Sapir, 2015) is likely to have evolved by pollinator-mediated selection acting primarily on floral colours to mimic large achromatic (i.e. 'bee-black') protective shelters (Vereecken et al., 2013). Finally, our in situ experiments on pollinator preferences also demonstrated that pollinator visits in the Oncocyclus irises are not motivated by an increased morning floral heat reward in tunnels facing the rising sun (Vereecken et al., 2013). This is also supported by a series of studies on the thermoregulatory abilities of wild bees illustrating that a 2 to $4^{\circ} \mathrm{C}$ increase of heat within flowers might be regarded as meaningless in light of the very high body warm-up rates of most medium-to-large vernal solitary bees recorded as pollinators of the Oncocyclus irises (Stone \& 
Willmer, 1989; Heinrich, 1993; Willmer \& Stone, 2004). Lavi \& Sapir (2015) have not touched upon this research, and in their article they focus only upon 'morning floral heat' as a reward for pollinators (see their first sentence of the Discussion section, 'Pollinators of the Oncocyclus irises are attracted to the heat absorbed in the dark-colored flowers in the morning (Sapir et al., 2006)'), a theory that is not statistically supported by any direct evidence in these irises (only indirect, correlative evidence with small sample sizes was provided; Vereecken et al., 2013).

Finally, Lavi \& Sapir (2015) note in their methods section that: 'These [Iris] species are completely self-incompatible, and maternal fitness (fruit and seed production) depends entirely on the nightsheltering pollinators (Sapir etal., 2005; Watts et al., 2013)'. However, Watts et al. (2013, p. 395) note explicitly that: '[...] Honey bees were found to be frequent diurnal visitors; they removed large quantities of pollen and were as effective as male sheltering bees at pollinating this species'. Our personal field observations during 2010-2011 suggest that the Netanya Iris Reserve where Lavi \& Sapir (2015) conducted their study on Iris atropurpurea was not devoid of honey bees, which could have bearing on the interpretation of the results presented by Lavi \& Sapir (2015).

We regret that Lavi \& Sapir (2015) failed to build on the evergrowing body of scientific knowledge on the quantification of selection gradients driving the evolution of floral traits, and that they did not use the mainstream analytical tools to study floral evolution (here, floral reflectance) from a pollinators' perspective. We think it would have been appropriate to discuss the most recent (and contradictory) evidence stemming from comparative phylogenetic studies on the evolution of floral traits (Vereecken et al., 2012) and from in situ measurements of pollinator preferences for floral scents or colours using state of the art methods (Vereecken et al., 2013).

In this Letter we highlight our recent work on a topic highly relevant to the work presented by Lavi \& Sapir (2015) (dealing with the same issues, in the same species, in the same biogeographical region) in order to provide the scientific community with a balanced view of contemporary research on the pollination of Oncocyclus irises. We encourage the community to adopt the use of modern methodologies that allow the analysis of floral traits from the pollinators' perspective. Only in this way will new light be shed on floral evolution in these and other fascinating examples of plant-pollinator interactions.

\section{Acknowledgements}

The authors are grateful to the two anonymous referees for their helpful comments on an earlier version of this Letter.

\section{Nicolas J. Vereecken ${ }^{1}$, Achik Dorchin ${ }^{2}$ and Amots Dafni ${ }^{3}$}

${ }^{1}$ Agroecology \& Pollination Group, Landscape Ecology \& Plant Production Systems, Université Libre de Bruxelles (ULB), Blvd. du Triomphe, CP 264/2, B-1050 Brussels, Belgium;
${ }^{2}$ USDA-ARS, Bee Biology \& Systematics Laboratory, Department of Biology, Utah State University, 5310 Old Main Hill, Logan, UT 84322-5310, USA;

${ }^{3}$ Department of Evolutionary and Environmental Biology, Institute of Evolution, Haifa University, Haifa, Israel (*Author for correspondence: tel +32 265068 44; email nicolas.vereecken@ulb.ac.be)

\section{References}

Arnold SEJ, Faruq S, Savolainen V, McOwan PW, Chittka L. 2010. FReD: the Floral Reflectance Database - a web portal for analyses of flower colour. PLoS ONE 5: e14287.

Chittka L. 1992. The color hexagon: a chromaticity diagram based on photoreceptor excitations as a generalized representation of colour opponency. Journal of Comparative Physiology A 170: 533-543.

Chittka L, Raine NE. 2006. Recognition of flowers by pollinators. Current Opinion in Plant Biology 9: 428-435.

Dyer AG, Whitney HM, Arnold SEJ, Glover BJ, Chittka L. 2006. Bees associate warmth with floral colour. Nature 442: 525.

Heinrich B. 1993. The hot-blooded insects: strategies and mechanisms of thermoregulation. Cambridge, MA, USA: Harvard University Press.

Hopkins R, Rausher MD. 2012. Pollinator-mediated selection on flower color allele drives reinforcement. Science 335: 1090-1092.

Lande R, Arnold SJ. 1983. The measurement of selection on correlated characters. Evolution 37: 1210-1226.

Lavi R, Sapir Y. 2015. Are pollinators the agents of selection for the extreme large size and dark color in Oncocyclus irises? New Phytologist 205: 369-377.

Mathew B. 1989. The Iris. London, UK: BT Batsford.

Papiorek S, Junker RR, Lunau K. 2014. Gloss, colour and grip: multifunctional epidermal cell shapes in bee- and bird-pollinated flowers. PLoS ONE 9: e112013.

Rausher MD. 1992. The measurement of selection on quantitative traits: biases due to environmental covariances between traits and fitness. Evolution 46: 616-626.

Sapir Y, Shmida A, Ne'eman G. 2005. Pollination of the Oncocyclus irises (Iris: Iridaceae) by night-sheltering male bees. Plant Biology 7: 417-424.

Sapir Y, Shmida A, Ne'eman G. 2006. Morning floral heat as a reward to the pollinators of the Oncocyclus irises. Oecologia 147: 53-59.

Sobel JM, Streisfeld MA. 2013. Flower color as a model system for studies of plant evo-devo. Frontiers in Plant Science 4: 321.

Spaethe J, Tautz J, Chittka L. 2001. Visual constraints in foraging bumblebees: flower size and color affect search time and flight behavior. Proceedings of the National Academy of Sciences, USA 98: 3898-3903.

Stone GN, Willmer PG. 1989. Warm-up rates and body temperatures in bees: the importance of body size, thermal regime and phylogeny. Journal of Experimental Biology 147: 303-328.

Vereecken NJ, Dorchin A, Dafni A, Hötling S, Schulz S, Watts S. 2013. A pollinators' eye view of a shelter mimicry system. Annals of Botany 111: $1155-1165$.

Vereecken NJ, Wilson CA, Hötling S, Schulz S, Banketov S, Mardulyn P. 2012. Pre-adaptations and the evolution of pollination by sexual deception: Cope's rule of specialisation revisited. Proceedings of the Royal Society of London B: Biological Sciences 279: 4786-4794.

Watts S, Sapir Y, Segal B, Dafni A. 2013. The endangered Iris atropurpurea (Iridaceae) in Israel: honey bees, night-sheltering male bees and female solitary bees as pollinators. Annals of Botany 111: 395-407.

Willmer PG, Stone GN. 2004. Behavioral, ecological, and physiological determinants of the activity patterns of bees. Advances in the Study of Behavior 34 $347-466$.

Key words: floral evolution, natural selection, pollination, shelter mimicry, wild bees. 